\title{
Bounds on the performance of rotated lattice constellations
}

\author{
Eva Bayer-Fluckiger \\ Institut de Mathématiques Bernoulli \\ EPFL 1015 Lausanne, Switzerland \\ eva.bayer@epfl.ch
}

\author{
Frédérique Oggier \\ Institut de Mathématiques Bernoulli \\ EPFL 1015 Lausanne, Switzerland \\ frederique.oggier@epfl.ch
}

\author{
Emanuele Viterbo ${ }^{1}$ \\ Politecnico di Torino \\ C. Duca degli Abruzzi 24, Torino, Italy \\ emanuele.viterbo@polito.it
}

\begin{abstract}
In this work, we give a bound on performance of any full-diversity lattice constellation constructed from algebraic number fields. We show that most of the already available constructions are almost optimal in the sense that any further improvement of the minimum product distance would lead to a negligeable coding gain.
\end{abstract}

Lattice constellations with high modulation diversity have been extensively studied as an alternative approach for transmission over the Rayleigh fading channel. The original idea was to introduce bandwidth efficient modulations with intrinsic diversity order and good minimum product distance to achieve substantial coding gains.

In $[2,3,1]$, it is shown that lattice constellations constructed using algebraic number theory provide the desired properties. The first examples using totally real algebraic number fields, yielding full-diversity, were given in [2]. Initially, no restriction on the shape of the lattice constellation was imposed, which resulted in either a complex bit labelling procedure or loss in the average energy. Further investigations were addressed to finding rotated $\mathbf{Z}^{n}$-lattices to avoid the above problems [5, 4]. In [1], several families of full-diversity rotated $n$-dimensional $\mathbf{Z}^{n}$-lattices from totally real algebraic number fields were given and analyzed for all dimensions.

The contribution of this work is to give a bound on the minimum product distance of any lattice constellation constructed from algebraic number fields. With the aid of this bound we are able to establish the ultimate coding gains achievable by such constellations. We show that most of the already available ones, constructed from totally real number fields, are within a few tenths of $\mathrm{dB}$ from the lower bound.

In the case of full diversity, the asymptotic coding gain of an $n$-dimensional lattice constellation $\Lambda$ is determined by the minimum product distance, [3]:

$$
d_{\mathrm{p}, \min }(\Lambda)=\min _{x, y \in \Lambda} \prod_{i=1}^{n}\left|x_{i}-y_{i}\right|=\min _{x \in \Lambda} \prod_{i=1}^{n}\left|x_{i}\right| .
$$

For high SNR, optimal lattice constellations achieve the maximum minimum product distance. In [1], it is shown that $d_{\mathrm{p}, \min }=1 / \sqrt{d_{K}}$, where $d_{K}$ is the discriminant of the number field $K$ over which we construct the algebraic lattice. Thus, maximizing $d_{\mathrm{p}, \min }$ is obviously equivalent to minimizing the field discriminant $d_{K}$.

A lower bound on the discriminant of number fields is given by Odlyzko [6]. The explicit computation of the lower bound is hard. Numerical tables for $n \leq 100$ are available for example in PARI [8]. Asymptotically, we have the following :

$$
\begin{aligned}
d_{K}^{1 / n} & =\left(4 \pi^{1+C}\right)^{r_{1} / n}\left(4 \pi e^{C}\right)^{2 r_{2} / n}-O\left(n^{-2 / 3}\right) \\
& \geq(60.8395 \ldots)^{r_{1} / n}(22.3816 \ldots)^{2 r_{2} / n}-O\left(n^{-2 / 3}\right)
\end{aligned}
$$

\footnotetext{
${ }^{1}$ This work was supported in part by CERCOM.
}

where $C=0.577215 \ldots$ is Euler's constant.

We compare the $d_{\mathrm{p}, \text { min }}$ of all best known constructions $[1,7]$ with Odlyzko's bound. The worst cases below dimension 30 appear for $n=7,13,17,19$ and 25, where the discriminant seems to be "far" from the bound.

Recall that the asymptotic coding gain ([3]) is given by:

$$
\gamma=10 \log _{10}\left(\frac{d_{\mathrm{p}, \min }(1)}{d_{\mathrm{p}, \min }(2)}\right)^{1 / n}[\mathrm{~dB}]
$$

where $d_{\mathrm{p}, \min }(i), i=1,2$ are the minimum product distances of two rotated lattice constellations with the same full diversity.

We compute the coding gain obtained using a number field whose discriminant would reach Odlyzko's bound relatively to the worst case constructions (see Table). We observe that the maximal gain would be at most $0.25 \mathrm{~dB}$. Hence, further improvement of the minimum product distance would lead to a negligeable coding gain.

\begin{tabular}{|c|c|}
\hline$n$ & coding gain \\
\hline 7 & 0.0301 \\
13 & 0.0855 \\
17 & 0.1179 \\
19 & 0.2064 \\
25 & 0.2461 \\
\hline
\end{tabular}

\section{REFERENCES}

[1] E. Bayer-Fluckiger, F. Oggier, E. Viterbo, "New algebraic constructions of rotated $\mathbf{Z}^{n}$-lattice constellations for the Rayleigh fading channel," IEEE Trans. on Inf. Theory, vol. 50, n. 4, pp. 702-714, April 2004.

[2] K. Boullé and J.C. Belfiore, "Modulation schemes designed for the Rayleigh channel," Proc. CISS'92, Princeton, NJ, pp. 288293, March 1992.

[3] J. Boutros, E. Viterbo, C. Rastello, and J.C. Belfiore, "Good Lattice Constellations for both Rayleigh Fading and Gaussian Channels," IEEE Transactions on Information Theory, vol. 42, n. 2, pp. 502-518, March 1996.

[4] J. Boutros and E. Viterbo, "Signal Space Diversity: a power and bandwidth efficient diversity technique for the Rayleigh fading channel," IEEE Transactions on Information Theory, vol. 44, n. 4, pp. 1453-1467, July 1998.

[5] X. Giraud, E. Boutillon, and J.C. Belfiore, "Algebraic tools to build modulation schemes for fading channels," IEEE Trans. on Inf. Theory, vol. 43, n. 3, pp. 938-952, May 1997.

[6] A. M. Odlyzko, "Bounds for discriminants and related estimates for class numbers, regulators and zeros of zeta functions: a survey of recent results," Séminaire de Théorie des Nombres, Bordeaux, pp. 1-15, 1989.

[7] F. Oggier, E. Bayer-Fluckiger, "Best rotated cubic lattice constellations for the Rayleigh fading channel," Proceedings of the IEEE International Symposium on Information Theory, 2003.

[8] PARI/GP-a software package for computer-aided number theory, C. Batut, K. Belabas, D. Bernardi, H. Cohen, M. Olivier. 